\title{
Using Open Source Software for Improving Dialog in Computer Science Education - Case Mozambique University
}

\author{
Nicholas B. Mavengere and Mikko J. Ruohonen \\ CIRCMI-Center for Research on Information, Customer and Innovation Management, \\ University of Tampere, Finland \\ Mikko.Ruohonen@uta.fi
}

\begin{abstract}
This paper highlights the essential matters in adopting technology as an aid to delivering education in higher education institutes in Africa. The key idea of the paper is how to use open source software for teaching and hence for improving interactivity between content, teachers and students i.e. how a wellselected open source software can foster learning processes. The paper also includes a case study in a Mozambican University to reveal a practical example on how technology has been used as an aid to education delivery. Availability of computer infrastructure, computer literacy, competent information technology staff, leadership support and collaboration and sharing culture are some of the identified issues important in using learning management systems (LMS) in African higher education institutes. The paper concludes by noting the need to fit the local environment and circumstances in adopting the use of technology in Africa.
\end{abstract}

Keywords: Learning Management Systems, Open Source Software, Higher Education Institutes, Africa.

\section{Introduction}

When IFIP Technical Committee 3 on Education had its first conference in a developing country in 1997 in Harare, Zimbabwe it was stated: "... while per capita income may be the dividing line separating "developed" from "developing" countries, the search for solutions to a host of common problems unites IT users around the globe. For example, IT adds a new level of complexity - acquiring equipment, training users and reconceptualising past practices - that places extraordinary demands on the IT community and, at the same time, creates dislocation in day-to-day life, whether in village or the megalopolis."(Marshall \& Ruohonen, 1998) The use of technology is of immense importance to the education sector. It is one of UNESCO's aims to ensure that both developing and developed countries have the best educational facilities essential to prepare youths to actively participate in modern society and add value to a knowledgeable society (Anderson et al., 2002). Information technology (IT) plays a very important role in this endeavor. However, Watson (1998) states, that "more attention is needed to consistent and appropriate pedagogies with an 
infrastructure for support that includes ease of access and time to incorporate IT into a well-established curriculum. Time for teachers to reflect professionally is essential. Strategies targeting professional capacity building will have a greater chance of success. They need to be focused not on the technology but on pedagogy and on the delivery mechanisms to be contexted within, rather than superimposed on, the real professional environment of the teacher."

Furthermore Visscher (2001) noted the general lag behind in use of computerized information systems in developing countries due to constraints for example in skills, expertise, finance and computer infrastructure. Nissilä and Puhakainen (2009) highlighted problems of technology related projects as including using expensive methods, ignoring local wishes, undermining local capacity and distorting priorities. The key idea of this paper is how to use open source software for dialogic teaching and hence for improving interactivity between content, teachers and students.

In addition the purpose of this paper is to highlight practical issues in educational management and systems in an African context. IT can be used to aid administration of a university i.e. to, for example, keep records of teachers and students. Another educational management perspective is classroom/teaching management which is handled by the teacher and is the focus of this paper. This educational management system has several names in the academic literature such as course management systems and learning management systems. While a significant number of tertiary education institutes in Africa have administrative educational management systems, there is even more room for improvement pertaining to systems that could be used for classroom. Moreover, seemingly the teachers' use of IT in their daily work is still a challenge (both in developed and developing countries). Beauchamp and Kennewell (2008) stated that "Most teachers adopting ICT use it for relatively authoritative teaching approaches, and our results suggest that they should identify how it can help achieve a more dialogic approach to whole-class teaching."

Furthermore, it has been noted that IS in the African educational sector are used mainly for administrative purposes with no direct impact on the main stakeholders in education i.e. students. Therefore, this research aims at motivating IT use in African universities in directly and positively impacting on learning methodologies. In addition, this research includes a case study from an African setting which will aid in providing experience in practical solutions on how to incorporate IT in enhancing education deliverance at tertiary level.

In this paragraph, we introduce potential learning management systems (LMS) which could be used for classroom management. This is important for awareness of existing platforms; some of them are part of free and open source software (FOSS) which African tertiary education institutes could use and benefit. The main advantage of using FOSS is the reduction of costs. It is important to highlight that the aim of this is to simply bring to light the possible LMSs. There is neither detailed discussion of these systems nor review of the systems in this paper. The first system KEWL (http://www.opensourceafrica.org/view_article.php?type=case_study\&id=16) is an African initiative and the second system Moodle (http://moodle.org/) is free, open source software and was used in the case study as elaborated in section 3. Other FOSS systems evaluated in the case study below (Section 3) are Dokeos (www.dokeos.com), Sakai (http://sakaiproject.org/portal) and ILIAS (www.ilias.de). 
The rest of this paper is structured as follows. Section 2 covers key issues in practical application of LMS taking into consideration the African setting. Next, the case study of the application of LMS in a Mozambican university is covered in section 3. Finally, the discussion in section 4 then highlights the key issues when implementing an LMS in Africa.

\section{Key Issues in Practical Application of LMS}

Kumar (2008) noted that technology incorporation in education is better than methods without IT use. However, there are several essential aspects that have to be taken into consideration for successful use of IT in education. This section discusses these aspects from an African perspective and in the tertiary education sector. These issues could be termed key issues in practical application of LMSs in African tertiary institutes. The issues that are discussed include computer literacy, computer infrastructure, collaboration/sharing culture, human resource (IT staff) and leadership support.

\subsection{Computer Infrastructure in Developing Countries}

The first requirement in using LMSs is the computer infrastructure. Kumar (2008) noted that the main challenge for IT-enhanced education in developing countries is availability of computer infrastructure. Wright (2006) highlighted that IT has long been recognized as having a key role to play in emerging markets but questioned the effort of African countries in promoting ICT for development. There is need for African countries to actively support and make initiatives to avail computer infrastructure to the education sector. Wright (2006) also notes that "the difficulty faced by Africa is one of access and in a global society increasingly driven by access to information Africa lags behind because its population does not have access to the technologies that are facilitating information access and dissemination."

\subsection{Computer Literacy}

Lecturers and students should be computer literate to be able to use LMSs. Kumar (2008) argued that this is a problem in use of IT in education in developing countries. There is need to incorporate IT literacy classes at teacher training institutions. There is also need to organize workshops to train in the use of IT. Students should also have compulsory computer literacy classes and be encouraged to make use of the computer in different aspects such as entertainment, research and assignments.

\subsection{Human Resource - IT Staff}

Anderson et al. (2002) suggested that countries must be able to benefit from technological development and to do so there is need for professionals with sound IT background. This IT background should not be computer hardware or software specific but general which enable learning and keeping in touch with technological advances. Different players in the tertiary education sector in Africa needs tailormade IT training which suits their job requirements. For instance there is need for 
trained IT staff who can manage the network on which the LMS runs. They should also have the essential skills to administer the LMSs. Different platforms such as Moodle and KEWL offer support to users which is an important aid in using the LMSs. The essential IT skills for running LMSs include basic knowledge on databases, network administration, web servers, system administration and server-side HTML embedded scripting language like PHP.

\subsection{Learning and Knowledge Sharing Culture}

The success of a LMS depends to a great extent on the need for collaboration at different levels. There are many benefits if there is collaboration from students, lecturers, departments, facilities and even between different universities. For instance in Finland a student can take courses from any university in the country. Libraries are open to both university personnel and other residents. This sharing culture and collaboration is essential especially to get the best out of limited resources. The power or usefulness of any LMS for example increases with the increase of users. And user value increases with the increase of input into the system. Hence the need for collaboration and sharing culture to effectively use LMS.

\subsection{Leadership Support}

The use of LMSs in Africa could be regarded as a change effort (Kumar, 2008) and change initiatives require the support of leaders This support has to be substantial and in some instances is recognized by resources put in support of these initiatives. A simple example is the inclusion of funds for IT in education in national budgets. Support at high levels such as in government should be cemented by policies which promote the use of IT in education. For instance computer literacy course could be mandatory at teacher training institutes.

\section{Case Study: Mozambican University Experience}

\subsection{Initial Findings}

The first intake in the Information Technology Department did a Database course in their third year of study. The Database course was done in two semesters. The first semester was taught using the "Traditional" face-to-face teaching methodology. There were many problems in the first semester, which are noted below.

At the end of the first semester, interviews were done with students to enquire about their Database course experience and below are some of their responses. From the interviews:

- "There was inadequate time to use the Module Resources."

- "It was difficult to communicate problems (academic and administrative) to the Lecturer."

- "It was difficult to seek help from other students." 
- "Sometimes we did not hear the deadlines set or forgot them when told long time before the date."

- "There was not enough time and space for discussions and debates."

- "We have inadequate or no practical experience with the use of Database especially in a System."

After the interviews an analysis of the problems was carried out and a review of the problems was done. This motivated research on how to improve class administration and the learning methodology.

\subsection{Problem Identification}

There was rampant shortage of study material for the database Module. Eighteen students and the lecturer relied on three main textbooks only. This made access to textbooks quite a problem and had adverse effects in the class performance and motivation. In addition, communication barriers were encountered in the first semester. There were no sufficient and efficient communication channels among the students themselves and between them and the lecturer. This made expression of problem areas and information dissemination quite difficult for the two parties. Furthermore, there were insufficient learning resources to carry out vivid discussions on the database concepts. This meant that the time each individual student had on the course material was limited. A conducive learning environment was difficult to create and this demotivated the students. And finally, there was lack of ICT hands-on-tools to improve the computer literacy of the pioneer class.

\subsection{Solution; Facilitating Learning by Blended Learning Approach}

After the problem analysis, the use of technology was recommended to aid face-toface learning and also help in the class administration. This led to consider blended learning approach (Colis \& Moonen 2001, see further discussion Kim 2007) which is used to describe learning that mixes event-based activities, including face-to-face classrooms, live e-learning, and self-paced learning. Research on how to incorporate technology was carried out and technology use was initiated to add to face-to-face learning methodology in the second semester. There were several possible FOSS alternatives that could be incorporated as described previously. However, there were several reasons that led to selection of Moodle such as stability, for it had a stable software platform at the time of the research, user support with well documented and free online support from users community, user-friendliness and personnel's expertise on its administrative and network requirements. Blended Learning was a combination of face-to-face learning and use of Moodle course management system (CMS) in the second semester. The following objectives were set for using a Blended Learning Approach in the second semester of the Database Modules:

- make Module materials available every time students need them

- improve communication between students and lecturer and among the students themselves 
- create space or platform for discussions and offer students alternatives in helping each other.

- improve computer literacy of the students.

The Database Module chapter materials were prepared and uploaded on the Moodle CMS before the chapter commences. The students then downloaded the materials for use. The materials were available on the university's local area network for the students to use. Face-to-face sessions were carried out using the materials downloaded. With Moodle CMS forums were utilised to discuss Database course concepts. Students uploaded their thoughts to Moodle CMS and debates were carried out which were accessible by students anytime of the day. This offered space for discussions and debates. Students also utilized this feature to highlight problem areas, difficulties and/or misunderstandings they had in the face-to-face sessions.

During face-to-face sessions students were informed about deadlines of tests, exercises and other activities. These deadlines were highlighted on the calendar, which was one of the features of Moodle CMS. Students were reminded every time they use Moodle CMS as the calendar is on the index page. Students also carried out case studies such as "The use of database systems in local Companies." These case studies were presented during face-to-face sessions. These Case Studies were carried out in group research settings and then uploaded on the Moodle CMS for further use.

\subsection{Evaluation of the Intervention}

The use of technology to improve the dialogic learning and interactivity effects had several outcomes. These outcomes can be described as technical, performance and social outcomes. In this section we will elaborate each of these outcomes.

\subsubsection{Technical Outcome; Diffusing New Technology}

Moodle CMS was the first information system to be used by the Information Technology Department Faculty of Tourism Management and Information Technology. It was an outstanding technical step for the Department as a whole. The Pioneer students participated in Moodle CMS set up. This enhanced their technology appreciation and knowledge. They also had the opportunity to practically learn MySQL database, PHP language, Apache web server and EasyPHP. In the first semester the students did not have any practical experience of Database use in a System. This made the grasp of Database Concepts difficult and this demotivated the students. In the second semester, Moodle CMS offered a platform to learn database (MySQL Database) use in a system. This motivated students to understand better the Database concepts.

\subsubsection{Performance Outcomes; Improving Overall Grades}

Figure 1 below shows the Database Module class performance in first semester and second semester respectively in the same student population. The graph shows the number of students in each mark range for the two semesters. 


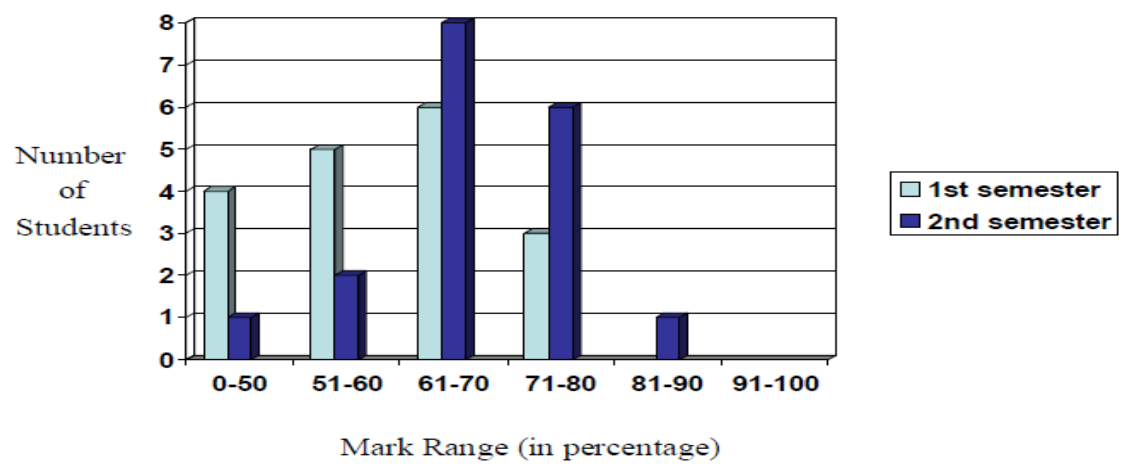

Fig. 1. Class performance in first and second semester

Four students failed in the first semester as compared to one in the second semester. Mark range 51-60 had three more students in first semester as compared to second semester. There were two more students in the mark range 61-70 in the second semester than in the first semester. This shows a positive shift from a lower range to an upper range, which is an improvement in performance of "average students". In the second semester there were six students in the mark range 71-80 and three students in the first semester (half of second semester). There was no student in the percentage range 81-90 in the first semester and one student in the second semester. This shows a better class performance in terms of both quality and quantity in the second semester.

\subsubsection{Social Outcomes; Increasing Motivation and Attendance}

Figure 2 below shows database course class attendance in first and second semester.

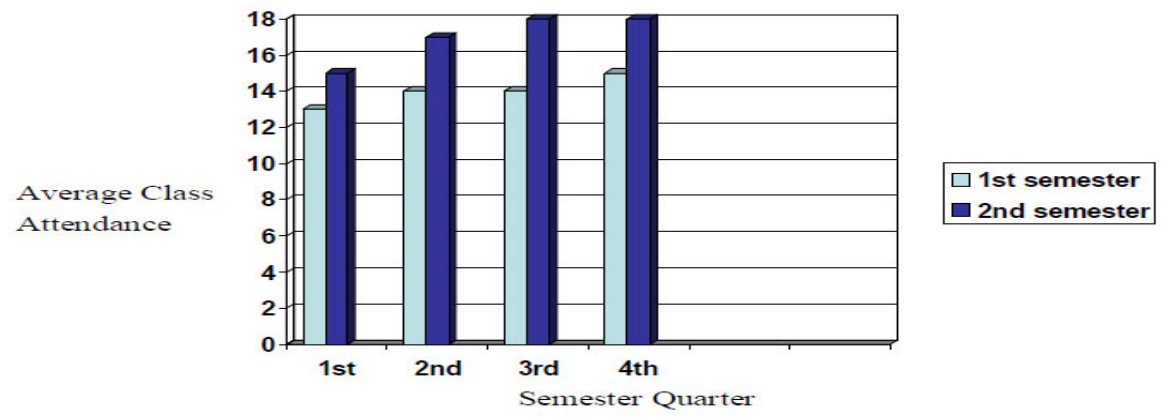

Fig. 2. Average class attendance in first and second semester

In the first quarter of the semester there was an increase in average class attendance by three students. The increase continued in the second quarter - we had an average class attendance of fourteen in the first semester and seventeen in the second semester. In the third and fourth quarters, there was full average class attendance in the second semester rising by four students in the third quarter and three students in 
the fourth quarter from the first semester. Moodle CMS shows statistics of usage by each user showing time logged on and out, last time accessed and frequency. The statistics revealed increased usage by the students each quarter from the first semester quarter to the last semester quarter. It also revealed high participation in discussion forums. This showed high interaction among the students and also with the lecturer. Students discussed socio-academic problems in forums and this motivated the students.

\subsubsection{Feedback from Interviews}

At the end of the second semester, interviews were done again with students to enquire about the impact of technological intervention to their learning experience and below are some of their responses.

- "Learning was fun with lots of material available that could be downloaded from the system."

- "There were lots of interaction through Moodle, which made problems be quickly addressed and from different perspectives of other students and the lecturer."

- "Studying with access to material (from Moodle) helped me understand better and improved my performance."

- "Technology use brought a new thinking in my life. It was like opening a window to a whole new world with possibilities."

- "Help as well as communication from other students and the lecturer improved significantly because of the new channel technology brought."

- "The system offered adequate space for discussions and debates."

We can state that the blended learning approach has had a great impact on student's learning skills, improved dialogic approach, increased motivation and better commitment to the use of educational management technology.

\subsection{Lessons Learned}

There was a significant improvement in performances as shown previously. This was attributed to a large extent, to the use of a blended learning approach. Hence the use of technology improved the performance of the Database course. There was improvement in computer literacy. Students maneuvered through the course management system, which was the first system they used. This triggered and encouraged students to start carrying out research in technology use in education. The use of Moodle CMS was an "eye-opener" and motivated students to study more.

The increase in class attendance shown was a result of the motivation and enthusiasm in use of technology by the class. Interaction among students increased significantly as noted by the number of emails they wrote each other. Discussion forums (on Moodle CMS) on academic, class administration and social issues were well participated by the students. Hence technology use had a positive social impact.

There is need for proper and good orientation in time before the implementation of blended learning. This is necessary to avoid panic and discomfort among students in using new technology. In addition, there is need to allocate adequate time for students' practice in using new technologies. For example, two students dismally 
dropped in performance and these were slow learners who did not grasp and adapt to use of technology very well. Remedial work is therefore recommended for the benefit of slow learners.

The main drawback and disadvantage of Moodle CMS use is when electronic materials are not available due to unavailability of network, electricity blackouts, server problems and/or the computer laboratory security rules. Continuous Moodle CMS access problems will raise adverse effects to class administration, progress and motivation. It is recommended that the responsible authorities and policy makers do appreciate and have technology use knowledge so that they fully support technology use.

Computer literacy is a prerequisite to use blended learning. However related to this, Nissilä and Puhakainen (2009) noted that one of the reasons for project failure is too much emphasis on technology and little attention to user needs. In addition, they highlighted the importance of including the users from the starting phases of the project. The success of blended learning in this case was due to the fact that the database course class was a computer literate group. It is therefore recommended that there be a computer literacy test before blended learning methodology use.

\section{Discussion}

Technology use in the tertiary education sector is of immense importance for educational development in Africa. The use of technology by higher education institutes varies, however this research focused on the LMSs. The key issues of learning management systems identified from an African tertiary education perspective are need for increasing computer literacy in the sector, need for computer infrastructure, learning and knowledge sharing culture, human resource - IT staff and leadership support. This paper included a case study of how open source software was utilized to benefit students at a university in Mozambique. This case demonstrated that there is no defined way of technological adoption but there is need to adjust and adapt technology to suit the different local conditions and circumstances. Nevertheless the use of technology is very much encouraged to aid and improve the higher education institutes in Africa.

\section{References}

Anderson, J., van Weert, T.: Information and Communication Technology in Education: A Curriculum for Schools and Programme of Teacher Development. In: UNESCO (2002), http: / / unesdoc . unesco.org/images/0012/001295/129538e.pdf

Beauchamp, G., Kennewell, S.: The Influence of ICT on the Interactivity of Teaching. Education and Information Technologies 13(4), 305-315 (2008)

Colis, B., Moonen, J.: Flexible Learning in a Digital World: Experiences and Expectations. Kogan-Page, London (2001)

Kim, W.: Towards a Definition and Methodology for Blended Learning. In: Fong, J., Wang, F.L. (eds.) Blended Learning. Workshop on Blended Learning 2007, Edinburgh, United Kingdom, Pearson, pp. 1-8 (2007)

Kumar, R.: Proceedings of World Academy of Science, Engineering and Technology, vol. 30, pp. 1307-6884 (2008) 
Marshall, G., Ruohonen, M.: Capacity Building for IT in Education in Developing Countries FIP Publication. Chapman \& Hall, London (1998)

Nissilä, J., Puhakainen, J.: Coopworks - A Case Study on an Information System Meant to Enhance the Capacities of Agricultural Co-operatives. In: International Conference on Social Implications of Computers in Developing Countries, Dubai, United Arab Emirates (2009)

Visscher, A.J., Wild, P., Fung, A.C.W. (eds.): Information Technology in Educational Management. Synthesis of Experience, Research and Future Perspectives on ComputerAssisted School Information Systems. Kluwer, Dordrecht (2001)

Watson, D.: Blame the Technocentric Artefact! What Research Tells us about Problems Inhibiting Teacher use of IT. In: Marshall, G., Ruohonen, M. (eds.) Op. Cit., pp. 185-191 (1998)

Wright, B.: The Role of ICT in African Development. African Business (325), 56-58 (2006) 